\title{
Color Performance, Durability and Handle of Inkjet-Printed and UV-Cured Photochromic Textiles for Multi-Colored Applications
}

\author{
Sina Seipel ${ }^{1 *}$, Junchun Yu ${ }^{1}$, Martina Viková ${ }^{2}$, Michal Vik $^{2}$, Marie Koldinskáa ${ }^{3}$, \\ Antonín Havelka ${ }^{3}$, and Vincent A. Nierstrasz ${ }^{1}$ \\ ${ }^{1}$ Textile Materials Technology, Department of Textile Technology, Faculty of Textiles, Engineering and Business, \\ University of Borås, Borås 50190, Sweden \\ ${ }^{2}$ Department of Material Engineering, Faculty of Textile Engineering, Technical University of Liberec, \\ Liberec 461 17, Czech Republic \\ ${ }^{3}$ Department of Clothing Technology, Faculty of Textile Engineering, Technical University of Liberec, \\ Liberec 461 17, Czech Republic
}

(Received October 31, 2018; Revised January 14, 2019; Accepted March 9, 2019)

This article is published with open access at Springerlink.com

\begin{abstract}
The development and design of novel functional and smart textile materials such as textile sensors and multicolored systems based on photochromic dyes necessitate controls of color intensities, switching speeds, and material durability. Precise control and synchronization of dye kinetics are important for multi-colored photochromic applications especially. However, durability towards abrasion and washing should not be compromised on if we aim to design reliable future textile products. In this study, two different commercial photochromic dyes - a naphthopyran and a spirooxazine-based dye - have been applied on PET fabric by inkjet printing and UV-LED curing. The photochromic textiles' color behavior, fastness to abrasion and washing, and handle are evaluated using spectrophotometry, scanning electron microscopy, and Kawabata evaluation system. Despite a decrease in color performance after washing, the photochromic inkjet print is effective and barely influences the textile structure. Reduced rigidity of the host matrix promoted higher color yields and faster dye kinetics, but also improved durability towards abrasion and washing. In order to synchronize kinetics of the different dye types for multi-colored applications, distinct curing conditions are preferable, which, however, result in varying print durability. In the design of multi-colored photochromic textiles, dye kinetics, and durability have to be balanced.
\end{abstract}

Keywords: Inkjet printing, UV curing, Textile sensor, Photochromic, Durability

(C) The Author(s) 2019, corrected publication 2019

\section{Introduction}

Although a limited number of products have reached the market, smart and functional textile high-end products have been an important topic for many years. In recent years, there has been an increasing interest in developing wearable, highly integrated technologies using resource-efficient processes [1]. Resource-efficiency, flexibility, and costeffectiveness play a key role in triggering innovation and promoting the production of smart and functional niche products like photochromic textiles among others. Combining inkjet printing and curing with UV-LED light facilitates a sustainable, flexible, and economic textile production and avoids the use of water, unnecessary amounts of chemicals, and energy while producing less waste. However, to the best of our knowledge, few studies on the resource-efficient production of photochromic textiles have been done. Aldib [2] explored inkjet printing of solvent-based photochromic inks and $\mathrm{Fu}$ et al. [3] investigated photonic curing.

What often is neglected is that the usage of novel technologies in the application of smart materials presents new challenges in the general behavior, durability and

*Corresponding author: sina.seipel@hb.se handle of functional textile materials. In general, inkjet printing and UV-curing are ideal technologies for functional multi-colored patterns because of their flexibility, costeffectiveness, and technical precision. Still, when combining different photochromic dyes in one product, it is important to synchronize coloration effects and fastness properties in order to provide consistent product quality. Therefore, we investigate the color behavior, fastness, and handle of multicolored photochromic textiles as seen in Figure 1(b), which are used in the design towards smart textile UV-sensors. A naphthopyran dye, Reversacol Ruby Red (RR), and a spirooxazine dye, Reversacol Sea Green (SG) are representatives of a more durable, but slow switching dye and a faster switching, but more temperature-dependent dye, respectively [4,5]. Both dyes are T-type photochromic compounds, which can reversibly switch color as a result of the absorption of electromagnetic radiation and thermally triggered reversion.

A common challenge in the application of photochromic dyes is their endurance in use, which is known as fatigue behavior. As a result of long-term UV-exposure and the influence of the predominant environmental factor oxygen, the color switching effect of photochromic compounds is weakened. Fatigue, which can also be referred to as photo- 

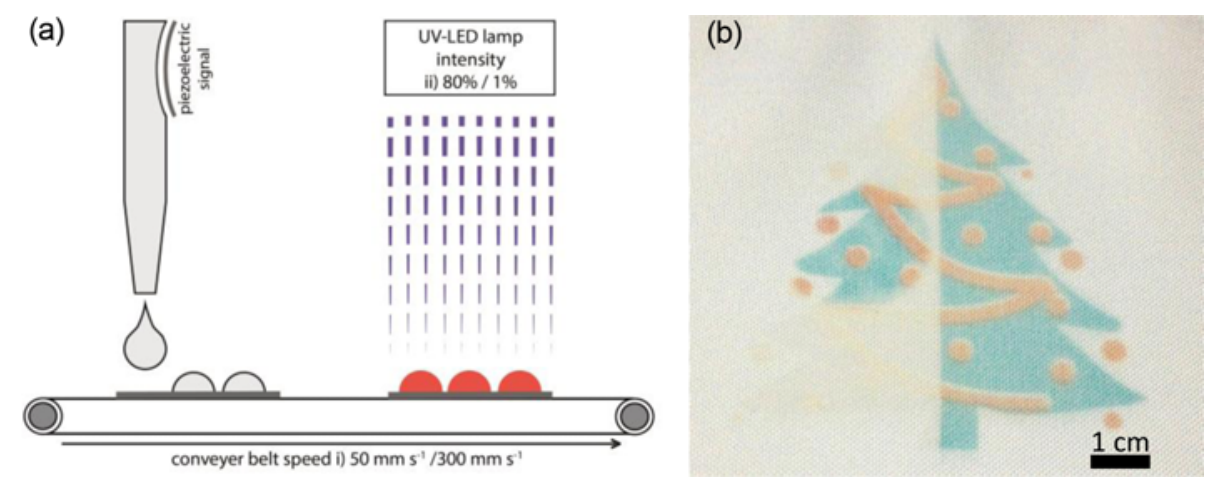

Figure 1. (a) Inkjet printing and UV-curing in a continuous production process using combined conveyor belt speeds (i) and lamp intensities (ii) of $50 \mathrm{~mm} / \mathrm{s}$ and $80 \%$ and $300 \mathrm{~mm} / \mathrm{s}$ and $1 \%$ and (b) partially activated multi-colored photochromic print on PET with RR and SG.

degradation, causes an irreversible chemical transformation where an undesired by-product is formed [6]. The formation of the fatigue product prevents the photochromic dye to revert to its original form, which prohibits further photoswitching [7]. Depending on the dye class and specific molecular modifications, photochromic dyes show varying photo-stability. Therefore, it is important to distinguish between fatigue of the dye and durability of the photochromic print as in its adhesion to the textile surface.

This study focuses on the durability of the photochromic prints produced and its implication on the design of a textile UV-sensor. The photochromic prints have been printed in single-pass mode and cured under strong and weak curing conditions, which means at a conveyor belt speed of $50 \mathrm{~mm} / \mathrm{s}$ combined with $80 \%$ of the maximum lamp intensity and $300 \mathrm{~mm} / \mathrm{s}$ combined with $1 \%$ of the maximum lamp intensity in a continuous pilot-scale inkjet printing system (Figure 1(a)). Seipel et al. [8] studied the interaction of a photochromic dye and ink matrix on molecular level to tune the kinetics of the photochromic reaction based on chosen fabrication parameters using inkjet printing and UV-curing. In that study, photochromic prints based on a naphthopyran dye, which are produced with a belt speed of $300 \mathrm{~mm} / \mathrm{s}$ and $1 \%$ lamp intensity, as well as $50 \mathrm{~mm} / \mathrm{s}$ combined with $80 \%$, indicated good wash fastness. Altering the viscosity and free volume of polymeric matrices to influence kinetics of photochromic dyes is an up-to-date topic. Dye kinetics in a host matrix can be influenced through different ways of polymer architecture by varying the type, dye placement, or $T_{g}$ of soft polymers [9-14], by the use of block copolymers [15], through the control of photo-polymerization $[8,16]$ and through pore dimensions in sol-gels [17]. In this study, however, we explore the effect of matrix rigidity on the durability of a spirooxazine and a naphthopyran-based print on textile via fastness tests, which generate mechanical action through abrasion and mechanical-chemical action at elevated temperature through washing. As textile sensors in use will be exposed to abrasion and washing, this investigation is necessary in regards to feasibility and design of future products for wearable or other textile sensor applications. Furthermore, the textile character and handle of the photochromic textiles are evaluated after intensive washing using a Kawabata evaluation system (KES) to determine the impact of the photochromic inkjet print on the textile structure.

We conclude that the choice of fabrication parameters of an industrially applicable process can modify dye kinetics, but also increase the durability of photochromic textiles via tuning the rigidity of the host matrix. The photochromic surface functionalization is effective without influencing the textile character. For multi-colored photochromic textiles, synchronization of dye kinetics and durability towards abrasion and washing have to be weighed against each other.

\section{Experimental}

\section{Materials}

Two types of photochromic dye were used in the formulation of UV-curable inkjet inks to produce UV-sensing textiles. The commercial heterocyclic spiro-compounds Reversacol Ruby Red and Reversacol Sea Green from Vivimed Labs, UK represent dyes of two different dye classes, naphthopyran and spirooxazine, respectively. The dye concentration in the designed inkjet inks was $4 \mathrm{~g} / l$. The UV-curable resin consists of dipropylene glycole diacrylate monomers (DPGDA), amine modified polyetheracrylate oligomers (Ebecryl 81) supplied by Allnex SA/NV, Belgium, and a UV-LED photoinitiator (Genocure TPO-L) supplied by Rahn AG, Switzerland. Solvents were used to disperse the photochromic dyes in the UV-curable resin, which were removed after stirring by vacuum pumping. For Ruby Red, ethyl acetate, 99.9\% (Chromasolv Plus) and for Sea Green, hexane, $\geq 97 \%$ (Chromasolv HPLC), both purchased from Sigma-Aldrich were used. The textile substrate for inkjet printing was a plain-woven polyester (PET) fabric with a weight of $147 \mathrm{~g} / \mathrm{m}^{2}$ and 20 and 23 threads/cm in warp and weft direction, respectively, received from FOV Fabrics, Sweden. 


\section{Production of Photochromic Prints}

UV-responsive smart textiles were produced by inkjet printing and UV-curing in a continuous process using a pilot-scale inkjet printing system with a conveyer belt for substrate transportation. Printing was carried out with a Starfire SA print head from Fujifilm Dimatix, USA at a resolution of 400 dpi in single-pass mode. The Starfire SA print head is able to print 4 different color scales, i.e. ink amounts. In this study, the darkest shade, i.e. the highest amount of a single pass with an effective ink amount of $16 \mathrm{~g} / \mathrm{m}^{2}$ was used. The print head temperature was set to $35{ }^{\circ} \mathrm{C}$ to facilitate ink flow upon printing. For curing, a UV-LED lamp FireJet from Phoseon Technology, USA with emission wavelength of 380 to $420 \mathrm{~nm}$ and a maximum emission power of $6 \mathrm{~W} / \mathrm{cm}^{2}$ was used. The photochromic prints were cured at two distinct conditions, which represent a high and low crosslinking density of the ink on the textile. Printing and curing was carried out at conveyer belt speeds of $50 \mathrm{~mm} / \mathrm{s}$ and $80 \%$ of the maximum lamp intensity (50_80) and at $300 \mathrm{~mm} / \mathrm{s}$ and $1 \%$ of the maximum lamp power (300_1).

\section{Fastness Testing of Photochromic Prints}

The fastness of the photochromic prints in terms of mechanical and chemical action was evaluated as a result of abrasion and washing. The photochromic prints were abraded using a Martindale tester according to ISO 12947:2006 with a weight of $9 \mathrm{kPa}$ during abrasion cycles of 5000, 10000, 15000 , and 20000 rubs. To determine the effect of washing on the print durability, samples were washed once and ten times after printing and curing. Washing was conducted according to ISO 6330:2012 using procedure 4N and reference detergent 3 . The samples were dried using drip flat drying.

\section{Color Analysis}

To evaluate the color behavior upon excitation (coloration) and relaxation (decoloration) of the photochromic prints an LCAM Photochrom 3 spectrophotometer was used. The specially designed device allows continuous color measurement

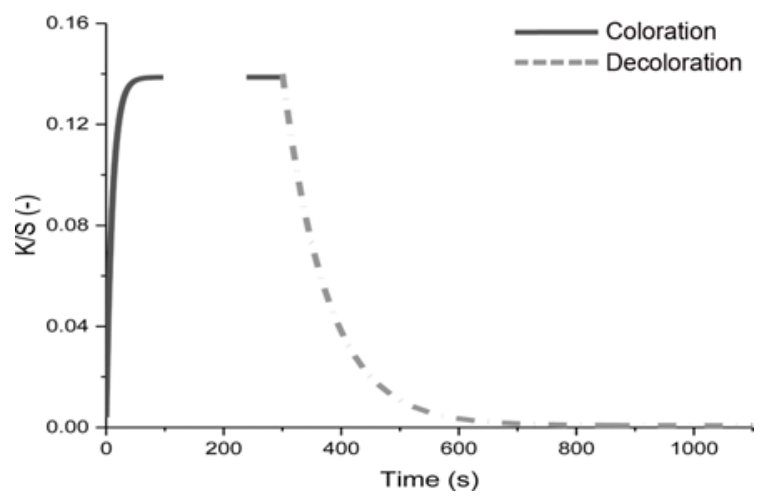

Figure 2. Scheme of color measurement cycle with $300 \mathrm{~s}$ of UVexposure for coloration and $800 \mathrm{~s}$ of relaxation for decoloration. upon cycles of UV-exposure and relaxation [18] (Figure 2).

As described previously, two distinct light sources were used for substrate activation and for color measurement [8]. An Edixon UV-LED lamp (EDEV-3LA1) with a radiometric power $\Phi_{\mathrm{V}}$ of $350 \mathrm{~mW}$ and an emission peak with wavelengths between $395 \mathrm{~nm}$ and $410 \mathrm{~nm}$ was used for activation. For measurement a dual light source system of combined high power white LEDs with CCTs of 4000, 5000, and $7000 \mathrm{~K}$ with sample illuminance of $60 \mathrm{klx}$ was used. Measured reflectance values $R$ are calculated to color values $K / S$ using the Kubelka-Munk equation.

$$
\frac{K}{S}=\frac{(1-R)^{2}}{2 R}
$$

The kinetic model shown in equation (2) follows firstorder kinetics and is generally used to describe photochromic color behavior for both the coloration and decoloration reaction as seen in Figure 2 [19].

$$
\Delta\left(\frac{K}{S}\right)=\left[\left(\frac{K}{S}\right)_{0}-\left(\frac{K}{S}\right)_{\infty}\right] \cdot e^{-k t}+\left(\frac{K}{S}\right)_{\infty}
$$

In the case of UV-curable materials, equation (2) is used for prints with a high degree of polymer crosslinking. For prints with lower polymer crosslinking density, equation (3) is used, which defines the kinetics of a decay mechanism as a result of continuous curing while color measurement and reconstructs the actual coloration curve [8].

$$
\Delta\left(\frac{K}{S}\right)_{c o l}=\left(\frac{K}{S}\right)_{\infty c o l} \cdot\left(1-e^{-k_{c o l} \cdot t}\right)-\left(\frac{K}{S}\right)_{\infty \text { dec }} \cdot\left(1-e^{-k_{d e c} \cdot t}\right)
$$

where, under the boundary condition that the processes of coloration and decay start simultaneously and at $\Delta K / S=0$, $K / S_{\infty c o l}$ is the maximum coloration value and $K / S_{\infty \text { dec }}$ is the final value of the decay reaction for each activation cycle. Kinetic rate constants for the coloration reaction and decay are defined as $k_{c o l}$ and $k_{d e c}$, respectively.

Based on the kinetic models, the performance of the textile UV-sensors was specified by its achieved color intensity $\Delta K / S$ upon activation with UV-light, its rate constant of color increase $k_{c o l}$ to achieve maximum coloration $K / S_{\infty}$, and its rate constant of color decrease $k_{\text {decol }}$ to revert to the initial colorless state $K / S_{0}$. The color performance of the photochromic prints was analyzed at each dye's reflectance minima. For prints containing naphthopyran dye Ruby red this refers to a wavelength of $500 \mathrm{~nm}$ and for those containing spirooxazine dye Sea green analysis was made at $620 \mathrm{~nm}$.

\section{Kawabata Evaluation System}

The change in handle of the plain-woven PET compared to after printing with Ruby red ink and after washing for 10 cycles was objectively analyzed using a Kawabata evaluation system (KES). The samples with dimensions of $200 \times 200 \mathrm{~mm}$ were printed and cured as three parallel stripes and measured both in warp and weft direction using a KES. KES measures 
properties of textile fabrics and predicts the aesthetic quantities perceived by human touch. The primary hand value (HV) and the total hand value (THV) were calculated based on tensile, shear, bending, compression, and surface properties of the fabrics. To categorize the non-skin contact, wearable textile UV-sensor, a men's summer suit was chosen as reference application for the KES-test. Here, the HV of the samples is based on evaluated stiffness (koshi), crispness (shari), fullness and softness (fukurami), and anti-drape stiffness (hari) of the fabrics on a scale between 1-10, where 1 stands for weak and 10 for strong properties. THV is evaluated using a scaling system between $0-5$, where 0 stands for out of use and 5 for an excellent fit of the measured material for the application.

\section{Scanning Electron Microscopy}

Scanning electron microscopy (SEM) was performed using a JEOL JSM-6301F instrument. Images were taken at an acceleration voltage of $6 \mathrm{kV}$ and a working distance of $39 \mathrm{~mm}$. To improve sample conductivity prior to SEM analysis the substrates were sputtered with a $3 \mathrm{~nm}$ thick layer of gold.

\section{Results and Discussion}

\section{Fitting of the Color Data of Prints Produced at $300 \mathrm{~mm} / \mathrm{s}$ and $1 \%$}

The extended model according to equation (3) is a mathematical approach to fit the color data of UV-curable photochromic materials with a low polymer crosslinking density. Based on the measured $K / S$ values of a photochromic dye, the coloration curve is fitted while taking a decay mechanism upon color measurement during UV-exposure and resulting activation into account. Equation (3) is a valid model for printed and abraded samples, which exhibit decay during color measurement. The fitted coloration curve of printed RR (Figure 3(a)) reaches a $\Delta K / S$ of 0.27 , whereas after abrasion with 20000 rubs (Figure 3(b)), $\Delta K / S$ is lowered to 0.2. However, when fitting the color data of SG prints, $\Delta K / S$ of the printed sample (Figure 3(c)) with a value of 0.06 is lower than after abrasion with $\Delta K / S$ of 0.1 (Figure $3(d))$. This repeated observation for all SG prints, which are cured at $300 \mathrm{~mm} / \mathrm{s}$ belt speed and $1 \%$ lamp intensity, shows that abrasion provides a loosening effect in the matrix and therewith less rigidity and more space for the dye molecule to undergo isomerization. A factor of influence on the distinct dye-matrix interaction of SG prints could be that spirooxazines have a large dipole moment [20]. A large dipole moment increases interactions of the dye molecule with the surrounding matrix and hence affects dye kinetics. Larkowska et al. [21] observed that the relaxation reaction appeared to be slower when the dipolar interactions were more pronounced. Therefore, the large dipole moment of the spirooxazine dye molecules and their consequent higher polarity during isomerization may influence their behavior in relation to the matrix more compared to RR prints.

\section{Factors of Influence on the Durability of Photochromic Prints}

Statistical analysis via a three-way ANOVA at a confidence

(b)
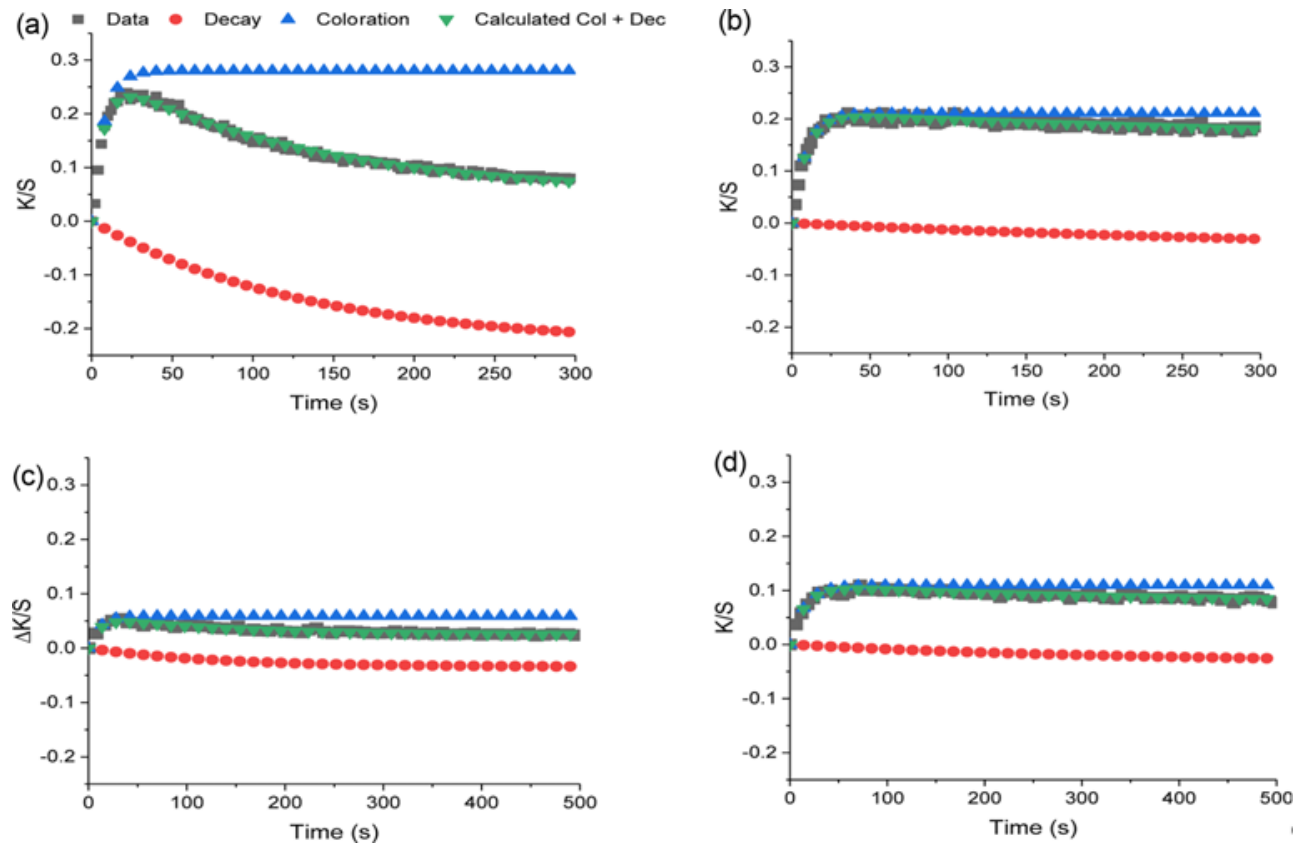

Figure 3. Fitting of $K / \mathrm{S}$ data of prints cured at $300 \mathrm{~mm} / \mathrm{s}+1 \%$ (a) RR printed, (b) RR abraded with 20000 rubs, (c) SG printed, and (d) SG abraded with 20000 rubs. 


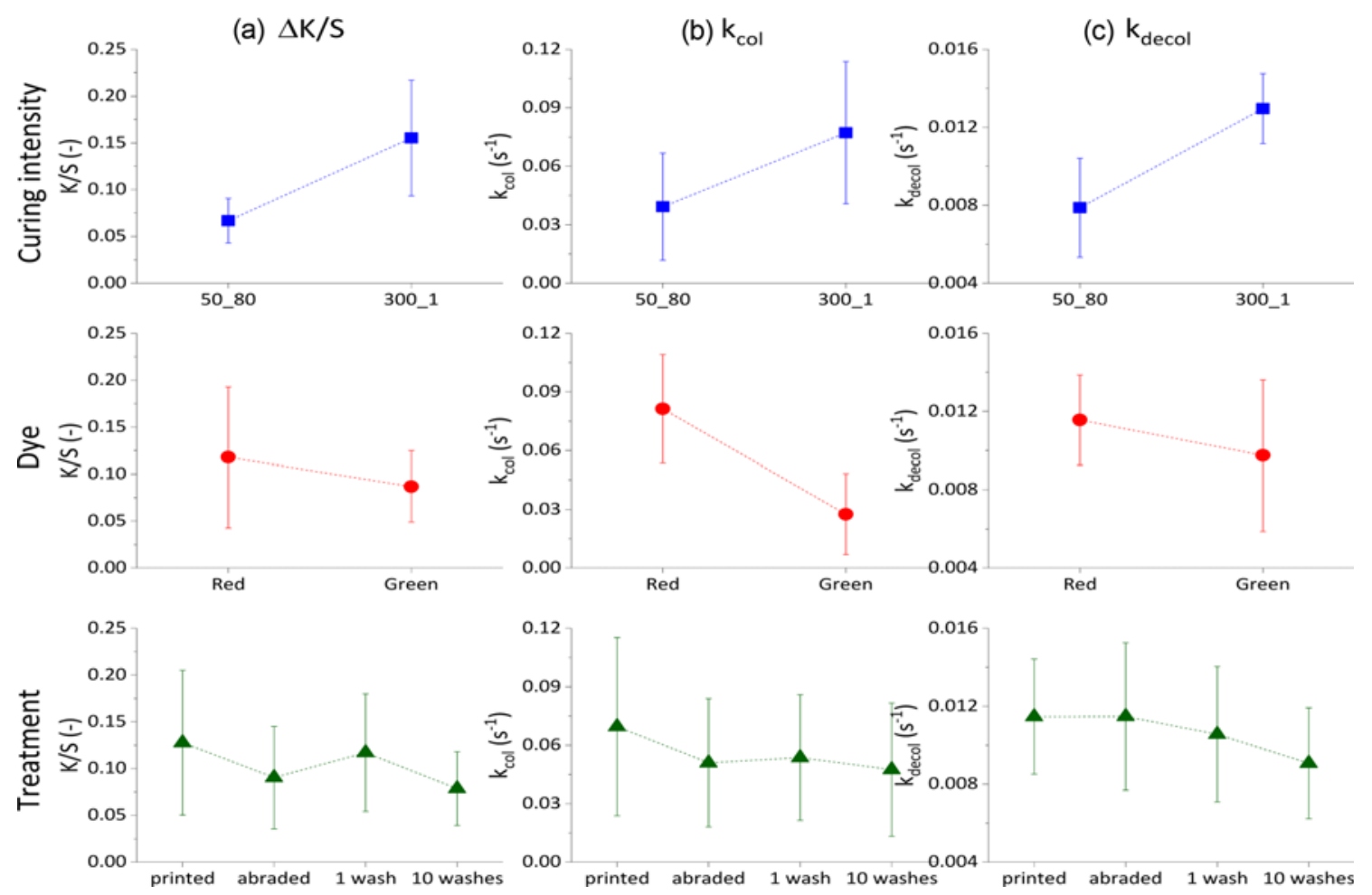

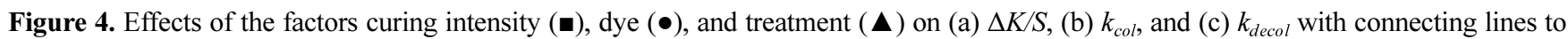
guide the eyes.

interval of $95 \%$ showed that the factors, curing intensity, dye, and treatment have significant effects on the color performance of photochromic prints in terms of the color yield $\Delta K / S$ and the reaction rates $k_{c o l}$ and $k_{\text {decol }}$. Curing intensity refers to a combined belt speed and lamp intensity of $50 \mathrm{~mm} / \mathrm{s}$ and $80 \%(50 \quad 80)$ and $300 \mathrm{~mm} / \mathrm{s}$ and $1 \%$ (300_1), which represent a high and a low curing intensity, respectively. Dye refers to naphthopyran dye Ruby red (RR) and spirooxazine dye Sea green (SG). Treatment includes printed, abraded with 20000 cycles, $1 \times$ washed, and $10 \times$ washed samples. As seen in Figure 4(a), prints cured with a low intensity $\left(\begin{array}{lll}300 & 1\end{array}\right)$ result in higher $\Delta K / S$ compared to curing with a high intensity (50_80) and red prints result in higher $\Delta K / S$ than green prints. Abrasion with 20000 cycles and 1 washing cycle have a similar effect, whereas 10 washing cycles reduce $\Delta K / S$ values by $50 \%$. The kinetics of the coloration reaction as seen in $k_{c o l}$ (Figure $\left.4(\mathrm{~b})\right)$ is higher for a low curing intensity (300_1), as well as for RR compared to SG.

Samples treated by abrasion, 1 wash, and 10 washes exhibit gradually decreasing $k_{c o l}$ as compared to printed samples. The same trend in regards to the effect of curing intensity, dye, and treatment is seen for $k_{\text {decol }}$ (Figure 4(c)). Higher $k_{\text {decol }}$ is achieved with higher belt speed and lower lamp power (300_1) and for RR dye. A gradual decrease in the decoloration kinetics is seen for abraded, $1 \times$ washed, and $10 \times$ washed prints.
Kinetics of photochromic dyes is affected by various properties of the surrounding media. Among these are the viscosity of the media, chain lengths of polymers, and specific placement of the dye molecules in the matrix [9,1113,15]. Seipel et al. [8] showed that adjusting the curing intensity of photochromic prints in an industrially applicable process changes the polymer crosslinking density of the UVresin. As a result of changed matrix rigidity, the dye-matrix interaction can be influenced and the kinetics of a photochromic textile controlled. Usually, spirooxazines are faster switching dyes than naphthopyrans [4]. Spirooxazines are more rigid with a higher molecular weight and more non-planar in structure in their colorless state [22]. However, upon isomerization spirooxazines become more or less planar and polar [23-25]. As can be seen in Figure 4 for the factor dye, RR disposes of stronger $\Delta K / S$ and higher switching rates $k_{c o l}$ and $k_{\text {decol }}$. Consequently, the integration of photochromic dyes in a UV-cured, cross-linked matrix largely affects their kinetics upon isomerization between the non-colored ring-closed and colored ring-opened states. The UV-cured, cross-linked matrix disturbs the isomerization of photochromic dyes between the non-planar and relatively non-polar form, i.e. the colorless state, and more or less planar and polar form, i.e. the colored state. In general, the factors polarity, free volume, rigidity, and direct interactions influence the photochromic response of materials [26,27].

Dye-matrix interactions are most pronounced for the 
colored form of photochromic dyes as the dyes change structure and polarity upon isomerization. The different treatments, which are differentiated in mechanical and chemical-mechanical action, affect the color performance of both dyes in different extent. Generally, in the factor analysis it is observed that mechanical action as in abrasion influences the color behavior less than chemical-mechanical action as in washing. In a study of Kert and Gorjanc [28], where cotton, PET, and a blend fabric was functionalized with commercial photochromic microcapsules in a pad-drycure process, fastness to washing, wet and dry cleaning, and wet and dry rubbing were tested among others. According to their study, dry rubbing (crock fastness) affected the color difference value $\Delta E$ of the samples less than washing. They conclude fastness properties to be a measure of adhesion of the binder to the fiber, in which the photochromic microcapsules are entrapped. Hence, compared to dry rubbing, washing has a harsher effect on the padded samples due to elevated temperature and combined mechanical and chemical action.

\section{Color Performance as a Result of Abrasion}

Via abrasion tests using a Martindale instrument photochromic prints undergo mechanical wear. As observed after consequent color analysis, mechanical action by various rubbing cycles (5000 to 20000 in 5000 steps) influences the photochromic prints on the textile surface compared to printed, i.e. non-abraded substrates. As seen in Figure 5(a) and (b), $\Delta K / S$ of SG prints is lower than for RR prints. However, the difference between a high and low crosslinking density of the ink resin on $\Delta K / S$ plays less role for $\mathrm{SG}$, the spirooxazine dye than for naphthopyran dye RR. SG prints with a low crosslinking density decrease in color yield from $\Delta K / S$ of 0.117 to 0.110 compared to the dye in a more rigid resin from $\Delta K / S$ of 0.085 to 0.063 . SG as the generically more rigid dye molecule might not have enough space to switch between its different isomers in neither the low nor highly cross-linked structure of the UV-resin. RR, however, is more flexible and therewith, larger differences in color yield can be observed. The difference in color kinetics observed in $k_{\text {col }}$ between the curing intensities 300_1 and $50 \_80$ is larger for SG $(5: 1)$ than for RR $(2: 1)$ as seen in average values for 5000 to 20000 rubs in Tables 1 and 2. As a result, it can be concluded that the rigidity of the UV-resin as a result of the curing intensity affects SG more than RR.

As previously mentioned, spirooxazines are generically faster switching dyes than naphthopyrans, but they also are more rigid in structure with a higher molecular weight and non-planarity in the colorless state than naphthopyrans $[4,22,29]$. If a dye that is generally fast-switching, but rigid

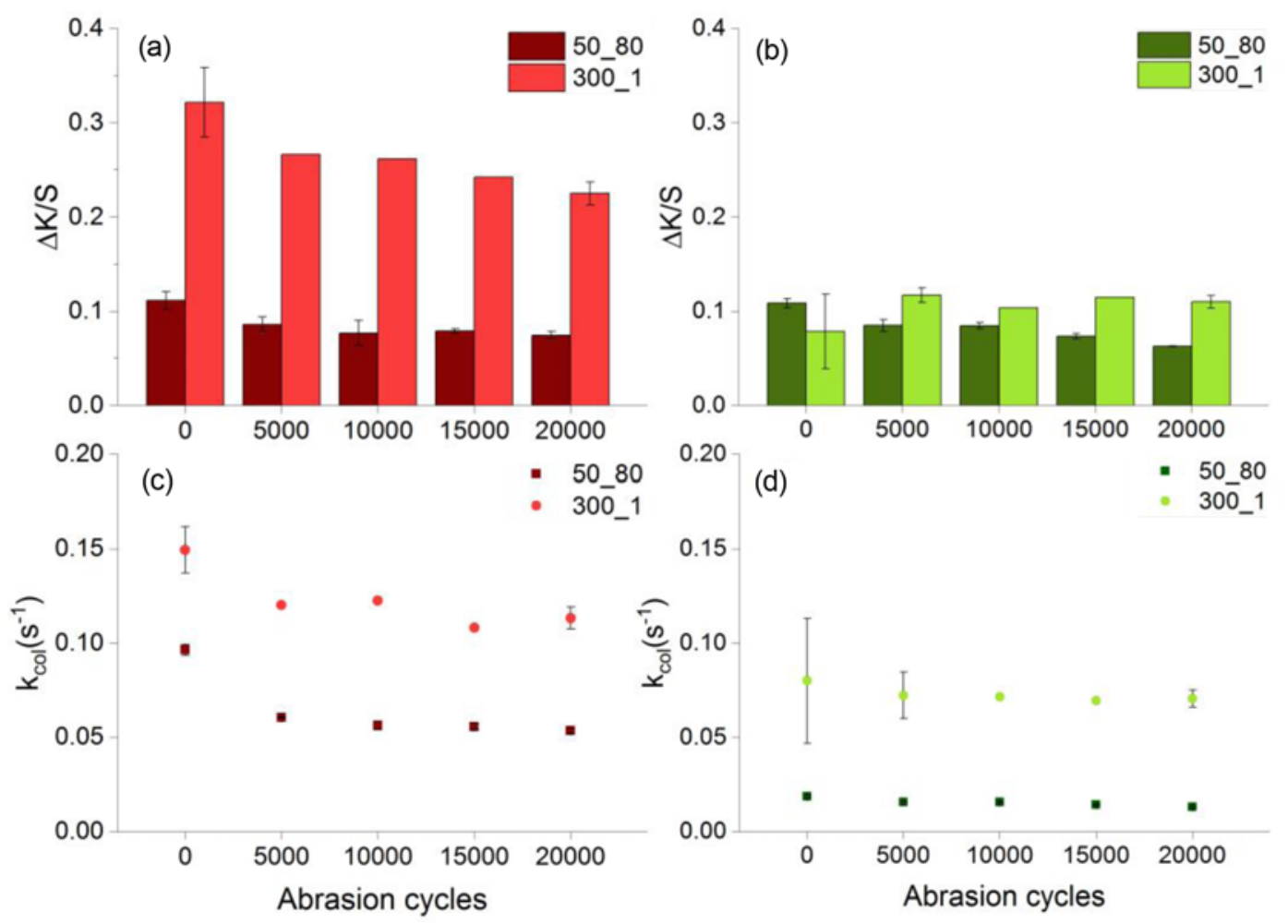

Figure 5. Effect of abrasion cycles 5000, 10000, 15000, and 20000 on $\Delta K / \mathrm{S}$ of RR (a) and SG (b) prints cured at $50 \mathrm{~mm} / \mathrm{s}$ and $80 \%\left(50 \_80\right)$ and $300 \mathrm{~mm} / \mathrm{s}$ and $1 \%\left(300 \_1\right)$ on and on $k_{c o l}$ of RR (c) and SG (d) prints cured at $50 \mathrm{~mm} / \mathrm{s}$ and $80 \%\left(50 \_80\right)$ and 300 mm/s and $1 \%$ (300_1). 
Table 1. Color yields $\Delta K / S$ and rate constants $k_{c o l}$ and $k_{\text {decol }}$ of RR prints printed, $1 \times$ and $10 \times$ washed and abraded with $5000,10000,15000$, and 20000 cycles

\begin{tabular}{|c|c|c|c|c|c|c|}
\hline & \multicolumn{3}{|c|}{$50 \mathrm{~mm} / \mathrm{s}+80 \%$} & \multicolumn{3}{|c|}{$300 \mathrm{~mm} / \mathrm{s}+1 \%$} \\
\hline & $\Delta K / S$ & $k_{c o l}$ & $k_{d e c o l}$ & $\Delta K / S$ & $k_{c o l}$ & $k_{d e c o l}$ \\
\hline Printed & $0.103 \pm 0.009$ & $0.089 \pm 0.007$ & $0.011 \pm 0.000$ & $0.235 \pm 0.068$ & $0.114 \pm 0.027$ & $0.014 \pm 0.002$ \\
\hline $1 \times$ washed & $0.062 \pm 0.002$ & $0.059 \pm 0.008$ & $0.009 \pm 0.000$ & $0.154 \pm 0.044$ & $0.102 \pm 0.007$ & $0.012 \pm 0.001$ \\
\hline $10 \times$ washed & $0.039 \pm 0.003$ & $0.048 \pm 0.009$ & $0.008 \pm 0.001$ & $0.136 \pm 0.005$ & $0.101 \pm 0.005$ & $0.013 \pm 0.001$ \\
\hline 5000 rubs & $0.086 \pm 0.008$ & $0.060 \pm 0.000$ & $0.010 \pm 0.000$ & 0.266 & 0.120 & 0.012 \\
\hline 10000 rubs & $0.077 \pm 0.013$ & $0.056 \pm 0.002$ & $0.010 \pm 0.000$ & 0.262 & 0.123 & 0.013 \\
\hline 15000 rubs & $0.079 \pm 0.002$ & $0.056 \pm 0.002$ & $0.010 \pm 0.001$ & 0.242 & 0.108 & 0.013 \\
\hline 20000 rubs & $0.075 \pm 0.004$ & $0.053 \pm 0.001$ & $0.009 \pm 0.000$ & $0.225 \pm 0.012$ & $0.113 \pm 0.006$ & $0.014 \pm 0.001$ \\
\hline
\end{tabular}

Table 2. Color yields $\Delta K / S$ and rate constants $k_{\text {col }}$ and $k_{\text {decol }}$ of SG prints printed, $1 \times$ and $10 \times$ washed and abraded with 5000, 10000, 15000, and 20000 cycles

\begin{tabular}{|c|c|c|c|c|c|c|}
\hline & \multicolumn{3}{|c|}{$50 \mathrm{~mm} / \mathrm{s}+80 \%$} & \multicolumn{3}{|c|}{$300 \mathrm{~mm} / \mathrm{s}+1 \%$} \\
\hline & $\Delta K / S$ & $k_{c o l}$ & $k_{\text {decol }}$ & $\Delta K / S$ & $k_{c o l}$ & $k_{\text {decol }}$ \\
\hline Printed & $0.107 \pm 0.002$ & $0.020 \pm 0.002$ & $0.008 \pm 0.001$ & $0.079 \pm 0.040$ & $0.080 \pm 0.033$ & $0.013 \pm 0.003$ \\
\hline $1 \times$ washed & $0.086 \pm 0.025$ & $0.017 \pm 0.004$ & $0.007 \pm 0.004$ & $0.153 \pm 0.011$ & $0.045 \pm 0.007$ & $0.014 \pm 0.001$ \\
\hline $10 \times$ washed & $0.050 \pm 0.006$ & $0.012 \pm 0.001$ & $0.005 \pm 0.000$ & $0.102 \pm 0.005$ & $0.030 \pm 0.002$ & $0.010 \pm 0.001$ \\
\hline 5000 rubs & $0.085 \pm 0.006$ & $0.016 \pm 0.000$ & $0.007 \pm 0.000$ & $0.117 \pm 0.008$ & $0.072 \pm 0.012$ & $0.019 \pm 0.010$ \\
\hline 10000 rubs & $0.085 \pm 0.003$ & $0.016 \pm 0.000$ & $0.007 \pm 0.000$ & 0.104 & 0.071 & $0.012 \pm 0.000$ \\
\hline 15000 rubs & $0.073 \pm 0.003$ & $0.014 \pm 0.000$ & $0.006 \pm 0.000$ & 0.115 & 0.069 & $0.013 \pm 0.002$ \\
\hline 20000 rubs & $0.063 \pm 0.000$ & $0.013 \pm 0.000$ & $0.007 \pm 0.004$ & $0.110 \pm 0.007$ & $0.071 \pm 0.005$ & $0.013 \pm 0.001$ \\
\hline
\end{tabular}

in structure is entrapped in a matrix with high rigidity, its degree of freedom is limited and slow kinetic rates can be expected. However, when the rigidity of the matrix is decreased, a larger effect of relative increase in $k_{c o l}$ can be observed for a fast-switching dye as a result of loosened dyematrix interaction. This can explain why the difference in photo-switching speed of SG prints is a 5-fold larger for 300 1 than for 5080 . The same change in matrix rigidity only affects RR prints with a 2-fold faster $k_{c o l}$ for $300 \_1$ compared to $50 \_80 . k_{c o l}$ of RR prints are decreased by $50 \%$ in a more rigid resin. For SG, an increased polymer crosslinking density of the matrix results in $20 \%$ of the initial $k_{c o l}$ compared to a softer matrix. SG prints seem to have a better abrasion resistance both observed in the color yield $\Delta K / S$ and the coloration rate $k_{c o l}$. Compared to RR prints, which show a steady decrease in $\Delta K / S$ and $k_{c o l}$ as function of abrasion cycles, $\mathrm{SG}$ prints are more stable. What can also be seen in $\Delta K / S$ values, which are presented in Tables 1 and 2 , is that the fastness properties are improved with increased matrix softness.

Whereas $\Delta K / S$ of $300 \_1 \mathrm{RR}$ prints are superior against abrasion compared to washing (Table 1 ), $\Delta K / S$ of $300 \_1 \mathrm{SG}$ prints show generally stable values for washed and abraded samples compared to in a more rigid matrix (Table 2). UVcurable coatings are in general known for their good durability and abrasion resistance [30-32]. Guan et al. [33] observed superior rubbing, wash, and color fastness of a UV-curable pigment ink compared to a commercial pigment ink, while the print softness was improved.

After 20000 abrasion cycles, RR prints 50_80 and 300_1 dispose of similar remaining color yield $\Delta K / \bar{S}$ with $70 \%$ and $67 \%$, respectively. However, the speed of the coloration reaction is influenced more pronounced for $50 \_80$ prints in regard to $k_{c o l}$ with remaining $55 \%$ of the initial rate constant compared to $300 \_1$ prints with $76 \%$ of the non-abraded value. SG prints, which have been abraded with 20000 rubs dispose of $58 \%$ of the initial $\Delta K / S$ for a rigid ink resin (50_80) and of $140 \%$ for a softer ink resin (300_1). After abrasion, the coloration rate $k_{\text {col }}$ of $50 \_80 \mathrm{SG}$ prints accounts for $70 \%$ of the initial rate and $88 \%$ for $300 \_1$ SG prints. As previously mentioned, the increase in color yield $\Delta K / S$ for $300 \_1$ SG prints is believed to result from a loosened matrix effect, which increases the mobility of the spirooxazine molecules and therewith the color intensity of the print.

\section{Color Performance as a Result of Washing}

The effect of washing on the photochromic prints is determined both after a single washing cycle and after extended washing of 10 cycles. Washing cycles have a significant effect on the color performance of RR and SG prints. The color yield $\Delta K / S$ of $\mathrm{RR}$ prints decreases gradually as a result of washing cycles. Decreasing $\Delta K / S$ of 
the naphthopyran dye is observed both when integrated in a matrix with high rigidity $\left(50 \_80\right)$ and in low rigidity (300 1). However, the absolute $\bar{\Delta} K / S$ with $0.103(50 \quad 80)$ is lower for prints with high polymer crosslinking density compared to prints with low crosslinking density with $\Delta K /$ $S=0.235$ (300_1) as seen in Table 1. Lower $\Delta K / S$ of prints with high matrix rigidity can be explained as a result of reduced space in the host matrix and hence limited degree of freedom for the dye upon isomerization.

In terms of durability, 300_1 RR prints dispose of $58 \%$ after 10 washing cycles compared to $50 \_80 \mathrm{RR}$ prints, which only have $38 \%$ remaining of the initial $\Delta K / S$. Although the fastness to washing for $3001 \mathrm{RR}$ prints is better than for $50 \_80 \mathrm{RR}$ prints, it should be noted that washing significantly affects the color yield of 3001 prints as compared to abrasion. Abrasion after 20000 revolutions barely shows an effect with $\Delta K / S=0.225$ compared to initial 0.235 . Washing after 10 cycles, however, results in a significantly lower $\Delta K / S=0.136$. Rate constants of the coloration reaction $k_{c o l}$ differ between 300_1 and 50_80 RR prints. Prints with a low degree of polymer crosslinking (300_1) show higher switching speeds with $k_{c o l}=0.1141 / \mathrm{s}$
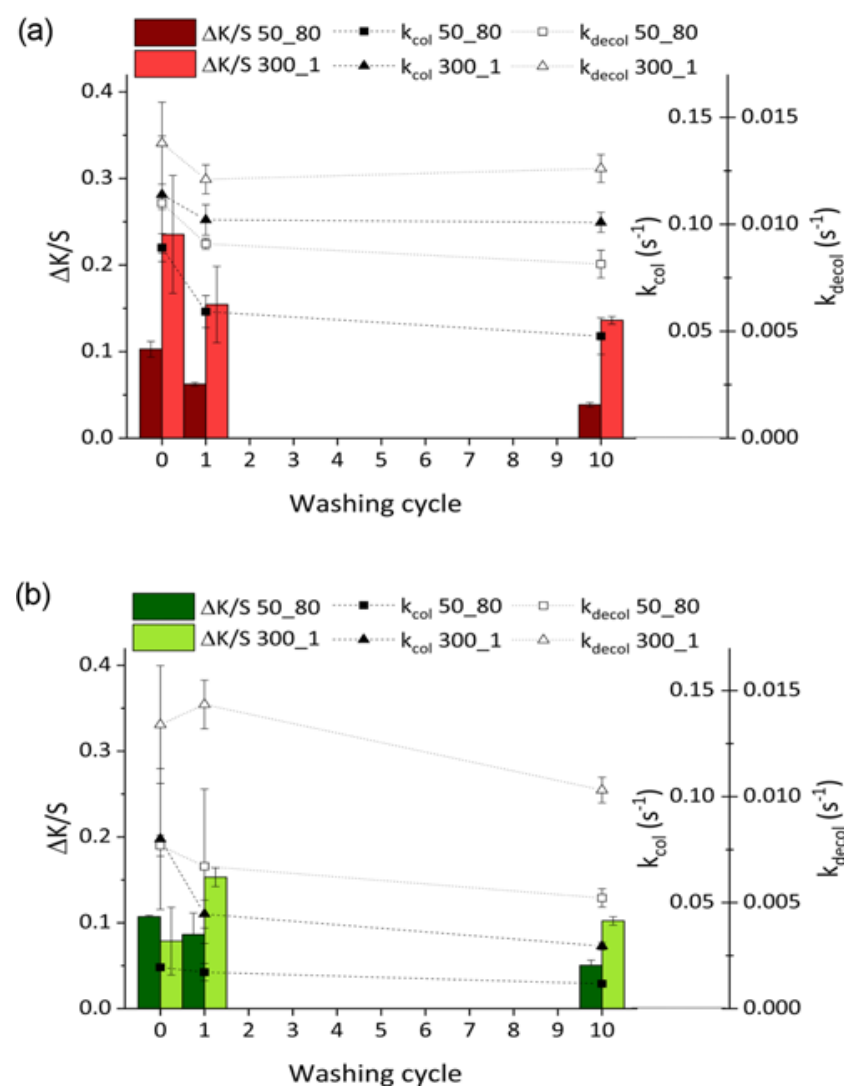

Figure 6. Effect of 0,1 , and 10 washing cycles on $\Delta K / S, k_{\text {col }}$, and $k_{\text {decol }}$ of (a) RR prints cured at $50 \mathrm{~mm} / \mathrm{s}$ and $80 \%(\square, \mathbf{\square}, \square)$ and $300 \mathrm{~mm} / \mathrm{s}$ and $1 \%(\square, \boldsymbol{\Delta}, \Delta)$ and of (b) SG prints cured at $50 \mathrm{~mm} / \mathrm{s}$ and $80 \%(\square, \mathbf{\square}, \square)$ and $300 \mathrm{~mm} / \mathrm{s}$ and $1 \%(\square, \boldsymbol{\Delta}, \Delta)$. compared to prints with high matrix rigidity $\left(\begin{array}{ll}50 & 80\end{array}\right)$ with a $k_{c o l}=0.0891 / \mathrm{s}$ of printed samples. After washing, rate constants are lowered for all RR prints. Whereas washing with different cycles gradually reduces the rate of photoswitching of $50 \_80$ prints from 0.089 to 0.054 to $0.048 \mathrm{1} / \mathrm{s}$, $300 \_1$ prints barely show a change between 1 and 10 washing cycles with $k_{c o l}$ of 0.102 and $0.1011 / \mathrm{s}$ (Figure 6(a)). Compared to the rate constant of printed samples, prints with low crosslinking density show superior fastness against washing with remaining $89 \%$ of the printed $k_{c o l} .50 \_80 \mathrm{RR}$ prints dispose of $54 \%$ the initial $k_{c o l}$. The speed of the decoloration reaction is approximately one order of magnitude slower than $k_{\text {col }}$ with $k_{\text {decol }}=0.0111 / \mathrm{s}$ and $0.0141 / \mathrm{s}$ of printed RR 50_80 and 300_1 samples, respectively. The same trend as function of washing cycles as for $k_{c o l}$ can be seen for rate constants under color reversion. Whereas $k_{\text {decol }}$ of $50 \_80$ prints decreases continuously after 1 to 10 washing cycles from 0.011 to 0.009 to $0.0081 / \mathrm{s}, k_{\text {decol }}$ of $300 \_1$ prints is stable after a single washing (Figure 6(a)). With $k_{\text {decol }}=0.0131 / \mathrm{s}$ after 10 washing cycles compared to the initial $k_{\text {decol }}$ of $0.0141 /$ s RR prints still dispose of $93 \%$ of the reversion rate. RR prints with high rigidity show remaining $73 \%$ after extensive washing. The degree of crosslinking of the UV-resin has a significant effect on the kinetics of the naphthopyran dye $\mathrm{RR}$, but also its durability as a result of washing. It can be concluded that a softer and more flexible host matrix, which gives more space for the dye upon isomerization, also promotes the fastness properties as a result of chemicalmechanical action.

The color performance of SG prints as a function of washing cycles differs from RR prints. In particular, color yield and kinetics of printed 300_1 samples deviate. As seen in Figure 6(b), $\Delta K / S$ of 300_1 printed, i.e. 0 washing cycles, with 0.079 is lower than after 1 washing cycle with 0.153 and also after 10 washing cycles with 0.102 . Washing gradually decreases color yields of SG prints with a high degree of polymer crosslinking from $\Delta K / S=0.107$ to 0.086 after 1 wash to 0.050 after 10 washes (Table 2). Whereas 5080 prints show remaining $47 \%$ of the initial color yield after extensive washing, 300_1 prints dispose of $129 \%$ of the initial $\Delta K / S$.

Little and Christie [4] have observed that an initial washing process increased coloration for spirooxazine in screen-prints, but then decreased coloration for further washing cycles. For naphthopyran dye in screen-prints the coloration was decreased directly for initial washing, as we also observe in RR prints. The initial color increase for spirooxazine was argued to be a consequence of a loosened binder matrix and hence facilitated isomerization. Since, spirooxazines are more rigid in structure than naphthopyrans, a change in rigidity of the host matrix has a large effect. Little and Christie [4] concluded that much of the behavior they observed was due to binder, not dye effects. Similarly, Billah et al. [22] found that a mild washing with an aqueous 
alkaline surfactant treatment facilitated the formation of the merocyanine form of a spirooxazine and thereby increased photochromic intensity.

Rate constants of the coloration reaction of prints with the spirooxazine dye differ depending on the curing intensity, i.e. the crosslinking density of the UV-resin. Same as for RR, 300_1 prints show faster photo-switching of printed SG with $k_{c o l}=0.0801 / \mathrm{s}$ than $50 \_80$ prints with $k_{c o l}=0.0201 / \mathrm{s}$. Washing affects the dye kinetics for both prints with high and low matrix rigidity. However, prints with low crosslinking density (300_1) are more affected with a loss in coloration rate of $62 \%$ after 10 washing cycles. Prints with high crosslinking density affect $k_{c o l}$ of SG with a $40 \%$ decrease. The decoloration reaction, which has approx. 3 times slower $k_{\text {decol }}$ compared $k_{c o l}$, is also significantly affected by washing. A continuous decrease in $k_{\text {decol }}$ is seen for $50 \_80 \mathrm{SG}$ prints from $0.008 \mathrm{1} / \mathrm{s}$ after printing to $0.007 \mathrm{l} /$ $\mathrm{s}$ after 1 wash and to $0.0051 / \mathrm{s}$ after 10 washes. Decoloration kinetics of SG in prints with low matrix rigidity (300_1) are unchanged after 1 washing cycle, but then decrease from 0.014 to $0.010 \mathrm{l} / \mathrm{s}$ after 10 washing cycles. This effect can also be a result of a loosened structure of the UV-resin upon initial washing, but then decreases due to extensive chemical-mechanical action after 10 washing cycles. In terms of fastness to washing, SG $50 \_80$ prints with $63 \%$ remaining $k_{\text {decol }}$ are inferior compared to $300 \_1$ prints with $77 \%$.

Maintenance and Repeatability of the Color Performance of Photochromic Prints

Repeatability of the photochromic color switching is an essential characteristic toward the design of a textile UVsensor in use. Hence, the color performance of $1 \times$ and $10 \times$ washed prints is assessed throughout several cycles of color activation and deactivation. Color measurement of RR prints with an applied ink amount of $19 \mathrm{~g} / \mathrm{m}^{2}$ cured at a belt speed of $300 \mathrm{~mm} / \mathrm{s}$ and $1 \%$ lamp intensity, show distinct behavior after 1 and 10 washing cycles. After a single washing process a continuous decrease in $\Delta K / S$ as function of activation and deactivation cycles is observed. However, prints after 10 washing cycles show stable and insignificantly different color yields $\Delta K / S$ and coloration, as well as decoloration rates over five and four activation cycles, respectively, compared to prints, which have been washed once (Figure 7). Periyasamy et al. [29] concluded that photo-degradation usually is higher during the initial cycles of UV-exposure due to a superficial layer of photochromic material, which degrades faster than material, which has penetrated further into fibers and fabrics. Our observation that more stable color performance is achieved after intensive washing, confirms the behavior. Therefore, we suggest that intensive washing should be considered as reduction process for UV-curable photochromic prints with low crosslinking density. Given that prerequisite, stable (a)

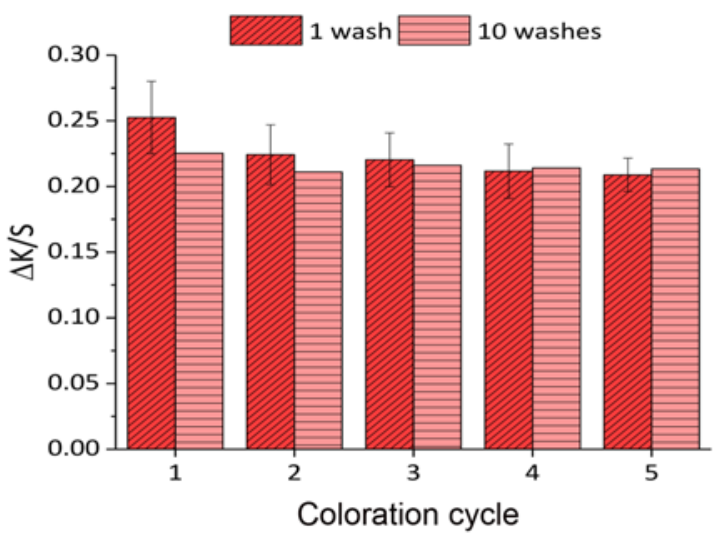

(b)
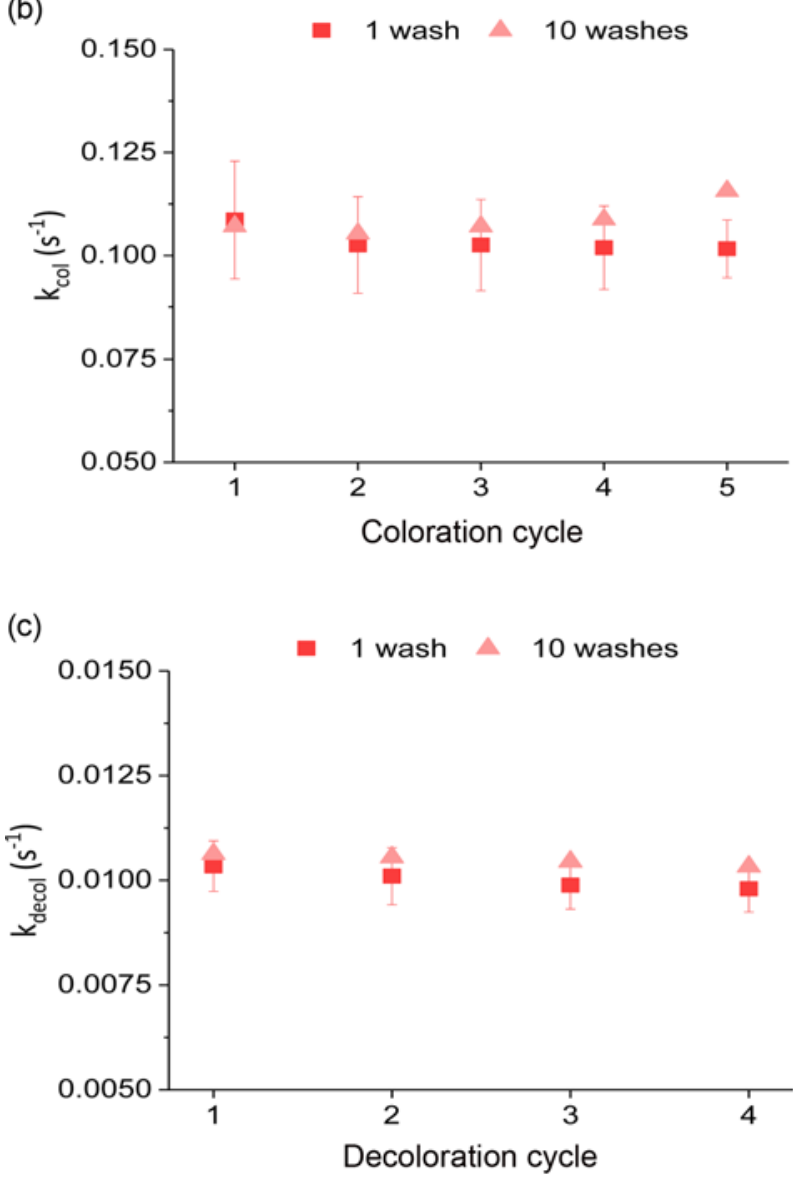

Figure 7. Comparison of $\Delta K / S_{c o l}$ (a), reaction rates $k_{c o l}(\mathrm{~b})$, and $k_{\text {decol }}(\mathrm{c})$ of prints with $19 \mathrm{~g} / \mathrm{m}^{2}$ of Ruby red ink cured at $300 \mathrm{~mm} / \mathrm{s}$ and $1 \%$ after 1 and 10 washes during 5 cycles of coloration and 4 cycles of decoloration.

color behavior is expected for long-term use.

\section{Effect of the Photochromic Print on the Fabric Structure and Handle}

KES analysis is made to objectively evaluate the handle of 
Table 3. HV including the single components and THV of the reference PET, printed and washed samples are tabulated. The functionalized samples were printed with $16 \mathrm{~g} / \mathrm{m}^{2}$ of ink, cured at a belt speed of $300 \mathrm{~mm} / \mathrm{s}$ and lamp intensity of $1 \%$, and then washed for 10 cycles

\begin{tabular}{cccccc}
\hline & \multicolumn{5}{c}{ HV } \\
\cline { 2 - 6 } & Koshi (stiffness) & Shari (crispness) & Fukurami (fullness and softness) & Hari (anti-drape stiffness) & THV \\
\hline PET reference & 8.14 & 4.45 & 3.68 & 10.27 & 1.60 \\
Printed & 8.37 & 5.73 & 3.22 & 10.55 & 1.84 \\
10× washed & 7.95 & 5.75 & 3.49 & 10.35 & 2.01 \\
\hline
\end{tabular}

the inkjet-printed PET fabric via measurement of mechanical properties. The results of the objective evaluation of the fabric handle show that the chosen application for the fabric was not ideal with a THV of 1.60 , which expresses a poor to below average fit. However, what can be seen is that after printing and washing the THV increases and therewith indicates a slightly better fit for the application. Important is the relative change of the fabric properties after printing and again after washing.

According to the stiffness values (koshi) in Table 3, it can be seen that the stiffness of the fabric increases after printing from 8.14 to 8.37 and decreases to 7.95 after 10 washing cycles. This shows that the stiffness of the fabric is in general high and increases due to the application of an ink layer. 10 washing cycles smoothen the printed fabric. The fabric crispness (shari) also increases after printing, but does not change after washing. The fabrics' fullness and softness, however, decreases slightly after functionalization with the photochromic ink, which is almost reversed again by 10 washing cycles. All measured samples show high anti-drape stiffness (hari) with values above 10 . The highest value with 10.55 is observed for the printed fabric and which is also slightly decreased again after washing.

Another essential property of a fabric, which is related to fabric comfort (softness and rigidity), is the bending moment. It quantifies the feeling by hand when bending the product. When bending the fabric under low-stress mechanical action, the comfort of the finished fabrics is assessed. The printed fabric has a bending moment $B=0.249$ $\mathrm{g} \cdot \mathrm{cm}^{2} / \mathrm{cm}$. After 10 washing cycles $B$ is reduced to 0.221 $\mathrm{g} \cdot \mathrm{cm}^{2} / \mathrm{cm}$. The larger the value of $B$ is, the harder is the object. Therefore according to the definition the printed
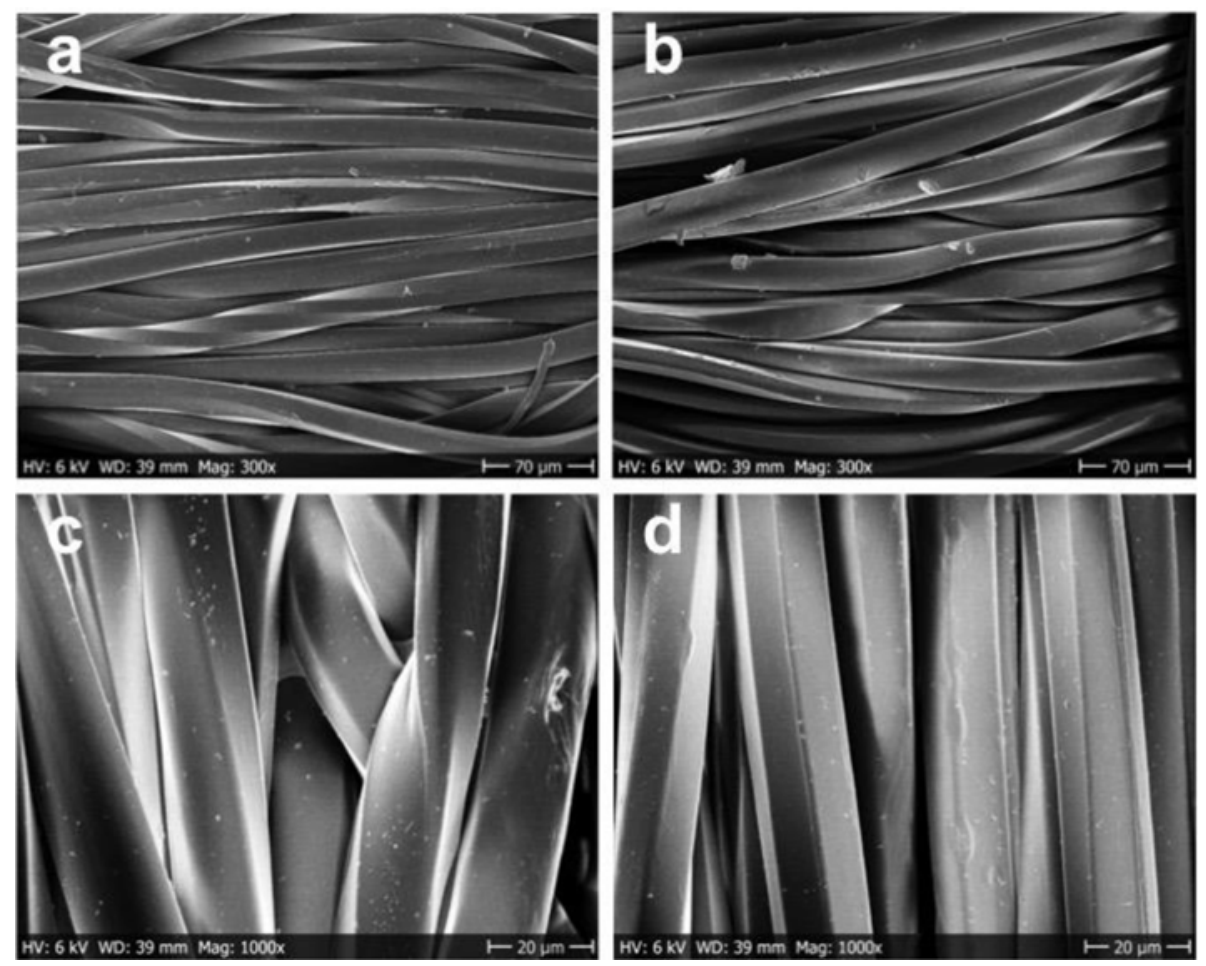

Figure 8. Printed PET samples with $19 \mathrm{~g} / \mathrm{m}^{2}$ of Ruby red ink after printing and curing at $100 \mathrm{~mm} / \mathrm{s}$ belt speed and $25 \%$ lamp intensity compared to reference PET; (a) printed weft multi-filament, (b) unprinted weft multi-filament, (c) printed warp multi-filament, and (d) unprinted warp multi-filament. 
photochromic textile is less comfortable and the washed photochromic textile is more comfortable than the nonprinted reference PET with $B=0.231 \mathrm{~g} \cdot \mathrm{cm}^{2} / \mathrm{cm}$. In summary, it can be said that KES-measurements confirm a stiffening effect of the fabric after printing, which is more or less revoked after 10 washing cycles despite preserved photochromic activity, which is seen in color measurements.

Also, observed changes of the fabric properties are low, which is attributed to the small amount of applied ink. In a research study of Karim et al. [32], where a UV-curable inkjet layer was applied on PLA fabric, the abrasion resistance of the fabric was improved while KES measurements showed that the ink layer did not significantly influence the handle of the fabric. Our study asserts their observation and hence that the photochromic surface functionalization of PET barely influences the textile handle of the plain-woven fabric. KES results are in line with the subjective handle when touching the fabrics, and are also supported by SEM analysis. As can be seen in Figure 8, the functional inkjetprinted material does not affect the fabric structure in terms of that the space between the fibers is not blocked. After printing with Ruby red ink (Figure 8(a) and (c)), the PET fabric does not show a difference compared to the reference fabric (Figure 8(b) and (d)). Therefore it can be expected that fabric properties like porosity and breathability are not affected. The pico-liter sized droplets, which are applied by ink jetting on the PET surface, are not detectable using SEM. Screen-printed fabrics typically show a change in surface structure, where the printing paste can clearly be detected on the fiber surface and pores between fibers are covered by the paste $[4,5]$. However, as is seen from the color data, inkjet-printed samples dispose of a photochromic color effect and hence prove that the ink adheres to the PET substrate without affecting the textile structure.

\section{Conclusion}

This study investigates important performance, durability and textile properties towards the production of a textile UVsensor using inkjet printing and UV-curing. With the aim to develop reliable textile sensors and multi-colored systems based on photochromic dyes, the controls of color intensities, switching speeds, and material durability in use are critical characteristics. In both cases, defined or even synchronized properties of different dyes are the pre-requisite for reliable future products. In this study, we showed that when combining the photochromic dyes Reversacol Ruby red and Sea green in one UV-cured smart textile, differences in kinetics and fastness towards abrasion and washing are to be expected. For spirooxazine-based UV-cured prints, color build-up will be slower and color yields lower than for naphthopyran-based dyes if prints are cured with the same intensity. Therefore, to synchronize dye kinetics with similar $k_{c o l}$ between 0.05 and $0.081 / \mathrm{s}$ RR should preferably be cured with a strong intensity and SG with a weak intensity. However, synchronization of dye kinetics brings about compromises in terms of weakened durability of RR prints towards washing and abrasion. If synchronization of dye kinetics can be compromised on, the choice of suitable fabrication parameters of an industrially applicable process can increase the durability of photochromic textiles exceeding the control of kinetics. Reduced rigidity of the host matrix promotes higher color yields and faster dye kinetics, but also improved fastness towards abrasion and washing. Mechanical action through abrasion has less impact on the color performance of the photochromic prints than mechanical-chemical action at elevated temperature through washing. Although washing affects the material on the textile, the photochromic activity of the inkjet print is preserved and barely influences the textile character. KES analysis shows that the textile handle is stiffer as a result of ink jetting and curing of the photochromic ink on the fabric surface, which, however, after 10 washing cycles is revoked. SEM analysis also indicates that the inkjet layer does not affect the textile structure in terms of its porosity and resulting breathability.

\section{Acknowledgements}

The authors are grateful for the support from Borås stad, TEKO (The Swedish Textile and Clothing Industries Association), Myfab and Sparbanksstiftelsen Sjuhärad for enabling this research.

Open Access: This article is distributed under the terms of the Creative Commons Attribution 4.0 International License (http://creativecommons.org/licenses/by/4.0/), which permits unrestricted use, distribution, and reproduction in any medium, provided you give appropriate credit to the original author(s) and the source, provide a link to the Creative Commons license, and indicate if changes were made.

\section{References}

1. C. Lang-Koetz, N. Pastewski, and H. Rohn, Chem. Eng. Technol., 33, 559 (2010).

2. M. Aldib, Color. Technol., 131, 172 (2015).

3. R. Fu, J. Shi, E. Forsythe, and M. Srour, Opt. Eng., 55, 124105 (2016).

4. A. F. Little and R. M. Christie, Color. Technol., 127, 275 (2011).

5. M. Viková, "Photochromic Textiles", Heriot-Watt University, Edinburgh, 2011.

6. J. C. Crano and R. J. Guglielmetti, "Organic Photochromic and Thermochromic Compounds", Springer, New York, 2006.

7. R. Pardo, M. Zayat, and D. Levy, J. Sol-Gel Sci. Technol., 
63, 400 (2012).

8. S. Seipel, J. Yu, A. P. Periyasamy, M. Viková, M. Vik, and V. A. Nierstrasz, RSC Adv., 8, 28395 (2018).

9. F. Ercole, N. Malic, S. Harrisson, T. P. Davis, and R. A. Evans, Macromolecules, 43, 249 (2010).

10. N. Malic, I. J. Dagley, and R. A. Evans, Dyes Pigment., 97, 162 (2013).

11. N. Malic, J. A. Campbell, and R. A. Evans, Macromolecules, 41, 1206 (2008).

12. G. Such, R. A. Evans, L. H. Yee, and T. P. Davis, J. Macromol. Sci., Polym. Rev., 43, 547 (2003).

13. R. A. Evans, T. L. Hanley, M. A. Skidmore, T. P. Davis, G. K. Such, L. H. Yee, G. E. Ball, and D. A. Lewis, Nat. Mater, 4, 249 (2005).

14. F. Ercole, T. P. Davis, and R. A. Evans, Macromolecules, 42, 1500 (2009).

15. K. Mutoh, Y. Kobayashi, and J. Abe, Dyes Pigments, 137, 307 (2017).

16. J. H. Kim, S. Y. Ban, Q. Zhang, G. W. Kim, M. J. Cho, and D. H. Choi, Mol. Cryst. Liq. Cryst., 445, 307/[597] (2006).

17. M. Parhizkar, Y. Zhao, Z. Xu, A. Gestos, Z. Xie, Z. Liu, and T. Lin, Fiber. Polym., 16, 2318 (2015).

18. M. Vik and M. Viková, "A Method and Device for Fatigue Testing of Photochromic, Fluorescent or Phosphorescent Dyes", Technicka Univerzita V Liberci, 2016.

19. M. Viková and M. Vik, Adv. Chem. Eng. Sci., 1, 224 (2011).
20. A. Ranjkesh, M.-K. Park, H. D. Park, J.-S. Park, J.-C. Choi, S.-H. Kim, and H.-R. Kim, Sensors, 16, 38 (2016).

21. M. Larkowska, M. Wuebbenhorst, and S. Kucharski, Int. J. Polym. Sci., 2011, 6 (2011).

22. S. M. R. Billah, R. M. Christie, and R. Shamey, Color. Technol., 124, 223 (2008).

23. M. Natali and S. Giordani, Chem. Soc. Rev., 41, 4010 (2012).

24. H. Xia, K. Xie, and G. Zou, Molecules, 22, 2236 (2017).

25. J. Zhou, Y. Li, Y. Tang, F. Zhao, X. Song, and E. Li, J. Photochem. Photobiol. A: Chem., 90, 117 (1995).

26. G. K. Such, R. A. Evans, and T. P. Davis, Macromolecules, 37, 9664 (2004).

27. X. Zhao, G. Wang, and K. Zhang, J. Appl. Polym. Sci., 124, 4157 (2011).

28. M. Kert and M. Gorjanc, Color. Technol., 133, 491 (2017).

29. A. P. Periyasamy, M. Vikova, and M. Vik, Text. Prog., 49, 53 (2017).

30. M. B. V. Shukla, D. K. Singh, and R. Shukla, Pigm. Resin Technol., 33, 272 (2004).

31. O. A. Hakeim, A. A. Arafa, M. K. Zahran, and L. A. W. Abdou, Colloids Surf. Physicochem. Eng. Aspects, 447, 172 (2014).

32. M. N. Karim, S. Afroj, M. Rigout, S. G. Yeates, and C. Carr, J. Mater. Sci., 50, 4576 (2015).

33. Y. Guan, B. Tawiah, L. Zhang, C. Du, and S. Fu, Colloids Surf. Physicochem. Eng. Aspects, 462, 90 (2014). 